Volume 2

\title{
The Role of Traditional Chinese Medicine in the Management of Chronic Pain: A Biopsychosocial Approach
}

John Burns

Tiffany A. Mullen

Follow this and additional works at: https://aah.org/jpcrr

Part of the Alternative and Complementary Medicine Commons, Integrative Medicine Commons, and the Therapeutics Commons

\section{Recommended Citation}

Burns J, Mullen TA. The role of traditional Chinese medicine in the management of chronic pain: a biopsychosocial approach. J Patient Cent Res Rev. 2015;2:192-196. doi: 10.17294/2330-0698.1206

Published quarterly by Midwest-based health system Advocate Aurora Health and indexed in PubMed Central, the Journal of Patient-Centered Research and Reviews (JPCRR) is an open access, peer-reviewed medical journal focused on disseminating scholarly works devoted to improving patient-centered care practices, health outcomes, and the patient experience. 


\title{
The Role of Traditional Chinese Medicine in the Management of Chronic Pain: A Biopsychosocial Approach
}

\author{
John Burns, DPT, MSOM, ${ }^{1}$ Tiffany A. Mullen, DO² \\ ${ }^{1}$ Acupuncture Clinical Services, Aurora Health Care, Milwaukee, WI \\ ${ }^{2}$ Department of Integrative Medicine, Aurora Health Care, Milwaukee, WI
}

\begin{abstract}
The National Institute of Medicine revealed that chronic pain affects more than 100 million adults in the United States, citing chronic pain as the leading reason patients seek medical care. Pain is also an extremely costly problem, with $\$ 635$ billion per year spent nationally, more than cancer, heart disease and diabetes combined. The biomedical model of chronic pain management has largely revolved around the use of narcotic analgesics for pain control. Unfortunately, this corresponds to a growth in the rate of abuse, misuse and overdose of these drugs. Additionally, there is an inherent failure rate to the myriad procedures used to control pain, such as spinal epidural injections and insertion of indwelling narcotic delivery systems, largely because these procedures fail to comprehensively address the multiple facets of pain generation. With its roots in the biopsychosocial model of pain management, traditional Chinese medicine may be a useful systematic or adjunct approach in the management of chronic pain. (J Patient-Centered Res Rev. 2015;2:192-196.)
\end{abstract}

Keywords chronic pain, biopsychosocial, traditional Chinese medicine, acupuncture, tai chi

A National Institute of Medicine report revealed that chronic pain affects more than 100 million adults in the United States. ${ }^{1}$ According to this report, chronic pain is the leading reason patients seek medical care with an estimated $\$ 635$ billion per year spent nationally. ${ }^{1}$ The cost of care for chronic pain exceeds that of cancer, heart disease and diabetes combined, largely because "the costs of unrelieved pain can result in longer hospital stays, increased rates of rehospitalization, increased outpatient visits, and decreased ability to function fully leading to lost income and insurance coverage." 2 Excessive costs aside, chronic pain exacts a more personal cost on the pain sufferer, that of risk of abuse, misuse and overdose of narcotic medications, a problem that has dramatically increased over the last several years. ${ }^{3}$ Despite use of narcotics as a mainstay of management of chronic pain in this country, it is well-documented that an "estimated $40 \%$ of patients with chronic pain do not achieve adequate pain relief." ${ }^{2}$ Similarly, invasive procedures such as spinal epidural steroid injections and implantable

Correspondence: John Burns, DPT, MSOM,

1020 N. 12th Street, 4th Floor, Milwaukee, WI, 53233,

T: 414-219-5910, F: 414-219-5914,

Email: john.burns@aurora.org time-release narcotic delivery devices not only haven't delivered consistent benefit to patients but also come at potentially significant risk to the patient., ${ }^{2,3}$

\section{Definition of Pain: Acute vs. Chronic}

The International Association for the Study of Pain defines pain as an "unpleasant sensory and emotional experience associated with actual or potential tissue damage, or described in terms of such damage." ${ }^{2}$ Pain can be further categorized as acute or chronic.

Acute Pain: Acute pain is a normal physiologic response, usually time-limited, to a noxious stimulus that enhances survival by warning the individual of impending or potential injury or progression of disease. If the noxious stimulus persists, changes in the peripheral tissues and both the central and peripheral nervous systems can lead to sensitization that worsens and prolongs the pain. Appropriate management of acute pain may prevent the onset of the pathophysiologic processes that change the spinal cord and brain and lead to chronic pain.

Chronic Pain: Chronic pain continues beyond the normal time expected for healing and is associated with the onset of pathophysiologic changes in the central nervous system that may adversely affect an individual's 
emotional and physical well-being, cognition, level of function and quality of life. Chronic pain serves no apparent useful purpose for the individual and may be diagnostically and therapeutically approached as a chronic disease process. The experience of pain, in general, is highly subjective and notoriously difficult to quantify or measure. For the purposes of this narrative review, we will focus only on the management of chronic pain.

\section{The Biopsychosocial Model}

Chronic pain is widely recognized as a biopsychosocial disorder. ${ }^{4,5}$ The biopsychosocial model is differentiated from the biomedical model in that the biopsychosocial model supports the concept that biological, psychological and social variables should be recognized as key factors in causing pain, disease and disabilities. ${ }^{6}$ To limit our treatment of pain to addressing only biological functions and biochemical mediators largely ignores one of the most disruptive features of pain that patients experience: emotional distress. $^{7}$

The influence of social influence (both positive and negative) on chronic pain management is also wellrecognized. For example, a study on family support and chronic pain was performed one year after patients completed an outpatient pain program. ${ }^{8}$ This study revealed that patients who reported having nonsupportive families used more pain descriptors when discussing their pain, relied more on medication and reported more pain sites. Conversely those patients who described their families as supportive reported having significantly less intensity of pain, greater activity levels and less reliance on medication.

Similarly, the doctor-patient relationship can influence the course of a pain condition. ${ }^{4}$ When patients perceive a physician as being supportive, they report decreased levels of distress. ${ }^{4}$ This relationship is often referred to as a "therapeutic alliance." Other health care providers can form therapeutic alliances as well. One study found that when there is a strong therapeutic alliance between physical therapists and patients with chronic low back pain, this supportive relationship was consistently a predictor of successful outcomes. ${ }^{9}$

\section{Traditional Chinese Medicine}

According to the National Institutes of Health, traditional Chinese medicine (TCM) incorporates a broad range of treatment practices that includes the relatively wellknown practices of both acupuncture and tai chi to treat or prevent health problems. ${ }^{10}$ TCM's view of health places less emphasis on anatomical structures and more emphasis on interactions, or relationships, among the body, mind, emotions and environment, or the social interactions of an individual. From this perspective, health is defined as a harmonious interaction of these entities and the outside world. The origin of TCM and the modalities of acupuncture and mind-body exercises date back more than 2,000 years. ${ }^{11}$ At the core of all TCM interventions is the appreciation that the physical and psychological aspects of each patient must be addressed to influence health and disease. ${ }^{11}$ Classical acupuncture is based on the theory that vital energy, called qi, flows through the body along pathways called meridians. There are specific points along these meridians, called acupuncture points, at which the qi may be accessed. Inserting needles into these points permits the practitioner to restore harmony to the system by rebalancing the flow of qi. ${ }^{12}$

In the biomedical model, acupuncture is thought to relieve pain through the gate-control mechanism or through the release of neurochemicals. ${ }^{13}$ Pomeranz and Berman ${ }^{13}$ described the possible neural mechanisms of acupuncture analgesia as follows: small-diameter muscle afferents are stimulated, sending impulses to the spinal cord, which then activates three centers (spinal cord, midbrain and pituitary) to release neurochemicals (endorphins and monoamines) that block pain messages. While acknowledging that there is some debate, Pomeranz and Berman concluded that the evidence supporting the endorphin hypothesis is very convincing. The authors asserted on the basis of supporting evidence from several studies that midbrain monoamines (serotonin and norepinephrine) are also involved in acupuncture analgesia; however, the role of the pituitary is less clear. ${ }^{13}$ Identifying possible mechanisms of action that fit within the paradigm of Western medicine has helped make acupuncture more acceptable in the United States.

The mind-body benefits of both acupuncture and tai chi are believed to be associated with their direct effects on the autonomic nervous system, which is involved in an individual's thoughts, emotions and behavior. ${ }^{11}$ From a TCM perspective, a person's emotional state, called shen in TCM, plays a crucial role in their behavior as 
well as a pivotal role in the development or exacerbation of disease and disability. ${ }^{11}$ This ancient understanding of the mind and body connection was addressed in the first line of an ancient text on utilizing acupuncture to alleviate pain, which stated: "The acupuncture method is rooted in the shen."14

\section{Evidence for TCM in Pain Management}

There are numerous studies that have demonstrated that TCM interventions, specifically acupuncture and tai chi, should be considered as viable modalities for treating chronic pain and emotional disorders. ${ }^{15,16}$ A meta-analysis of acupuncture's effectiveness on chronic pain concluded, "Acupuncture is effective for the treatment of chronic pain and is therefore a reasonable referral option." 17 Another meta-analysis was conducted on the use of auricular acupuncture to treat both acute and chronic pain conditions. Findings showed an overall significant improvement in pain scores for those patients receiving acupuncture versus controls. The authors concluded the evidence suggests that auricular acupuncture may be an effective treatment for chronic pain. ${ }^{18}$

Several studies on chronic pain and disability due to osteoarthritis also were evaluated. Osteoarthritis is the most common form of arthritis and the eighth leading cause of disability globally. ${ }^{19}$ In a meta-analysis of 10 randomized controlled trials with 1,456 participants, the authors concluded that "acupuncture is an effective treatment for pain and physical dysfunction associated with osteoarthritis of the knee."

Another important study compared acupuncture with "usual care" (medication and psychotherapy) for pain associated with depression that showed those patients treated with acupuncture did better than the usual care group. ${ }^{20}$ The findings showed that participants with moderate-to-extreme pain at baseline did better at 3 months if they received acupuncture (mean 6.0-point reduction from baseline 6.0 [95\% confidence interval (CI): 5.0-7.1] in Patient Health Questionnaire [PHQ-9] depression score and a mean 11.2-point reduction from baseline [95\% CI: 7.1-15.2) in the Short Form Health Survey [SF-36] bodily pain score 11.2) compared to the mean improvements seen in those who received counseling (4.3 [95\% CI: 3.3-5.4], 7.6 [95\% CI: 3.611.6], respectively) or usual care (2.7 [95\% CI: 1.50 4.0], 7.2 [95\% CI: $2.3-12.1$ ], respectively). ${ }^{20}$
Similarly, tai chi is recognized as a mind-body intervention that incorporates the principles of TCM. ${ }^{21}$ Tai chi is defined as an ancient martial art that is now widely practiced for its health benefits of improving physical and emotional well-being. ${ }^{22}$ According to a meta-analysis conducted by the Department of Veterans Affairs, the practice of tai chi was found to be an effective nonpharmacologic modality to reduce both pain ${ }^{23}$ and depression ${ }^{24}$ in veterans experiencing these conditions. Yet another meta-analysis suggested that practicing tai chi helped patients suffering from fibromyalgia in that it reduced their pain and improved both physical function and well-being. ${ }^{25}$ In the same study, a subanalysis of low back pain also showed that tai chi was beneficial for pain relief and disability in this population. ${ }^{25}$

As a movement therapy, tai chi is associated with promoting both physiological and psychological adaptations by modulating parasympathetic tone and the limbic system for improving functional abilities and for reducing emotional distress. ${ }^{21}$ These benefits are believed to be derived from tai chi's practice principles, which include meditation, visual imagery, slow rhythmic movements and coordinated breathing. ${ }^{24}$ This mind-body control modulates multiple aspects of health, including mood, pain and functions of the immune and peripheral autonomic nervous systems.

\section{Ancient Meets Modern: Combining the Biomedical Model with TCM}

There are several articles in the medical literature that examine the outcomes resulting from the use of both established biomedical models of chronic pain management and TCM treatment modalities. One metaanalysis compared management strategies for sciatica wherein the authors provided "new data to assist shared decision-making" with findings that supported the effectiveness of nonopioid medications, epidural injections, spinal manipulation and acupuncture. ${ }^{26}$ Another study addressed the use of acupuncture in the primary care setting alongside traditional approaches to chronic pain. This study suggested that acupuncture appeared to be a cost-effective clinically relevant intervention with "short- and long-term benefits for low back pain, knee osteoarthritis, chronic neck pain and headache. ${ }^{27}$ 
Knowing that all approaches to the management of chronic pain, whether biomedical or nontraditional (from a Western perspective), have some validity with respect to the literature, the most comprehensive approach to pain management we can offer patients may be to offer the best of both. In a clinical update by the International Association for the Study of Pain titled "Integrative Pain Medicine: A Holistic Model of Care," the author concluded the report by stating, "We cannot afford to ignore these realities: it is imperative that as health care providers we have an open mind to low-cost, low-risk integrative strategies that our patients are already embracing." 28

\section{Limitations}

There are many limitations in the area of TCM research. Both acupuncture and tai chi studies have an inherent problem to control for the placebo effect. ${ }^{24}$ A Cochrane review of acupuncture determined that due to patient bias and expectations to positive outcomes, the placebo effect could not be ruled out as a major contributing factor to the observed benefits associated with acupuncture interventions for a wide range of conditions. ${ }^{29}$ The use of "sham" acupuncture (the insertion of needles into points on the body not associated with any particular therapeutic effect) has been used as a means to circumvent this issue by providing a "control" for many acupuncture studies. It is also reported that tai chi studies similarly post a conundrum for researchers to discern specific versus nonspecific effects. ${ }^{30}$ Researchers have suggested that tai chi cannot be scientifically studied with a reductionist research framework that does not take into account the complexity of tai chi as a multicomponent intervention. ${ }^{31}$

Blinding also proves to be a difficult issue to solve in the TCM research literature. Of the nonspecific effects that are often underappreciated as contributing factors in treatment outcomes, within both research and clinical settings, is the recognition of importance of patient expectations ${ }^{32}$ and the therapeutic alliance developed between provider and patient. ${ }^{33}$ This new appreciation of patient expectations and the providerpatient relationship directly acknowledges the biopsychosocial model. When a patient's thoughts, emotions and beliefs (their shen) are addressed, treatment outcome and healing may be enhanced. ${ }^{15}$
This is germane to TCM practice. Accounting for this effect in research is confounding, making it difficult not only to "double-blind" the TCM practitioner, but to want to blind them at all in order to draw appropriate conclusions in the TCM paradigm.

Another limitation to the widespread adoption of TCM in the management of chronic pain is cost. While the delivery of TCM pain management is significantly less costly dollar-for-dollar compared with biomedical approaches, the cost nearly always falls to the patient. It is helpful that the Affordable Care Act (ACA) inserted language stating insurers may not discriminate with regards to the coverage of care offered by nontraditional versus traditional practitioners; ${ }^{34}$ however, this aspect of the ACA has yet to be legally implemented. With more attention to the efficacy of chronic pain management using less costly (to the health care system) options such as TCM continuing to appear in the medical literature, it is the hope of this review's authors that TCM will earn its place on the roster of covered benefits to all insured patients.

\section{Conclusions}

A major responsibility of medical practitioners of all kinds is to relieve suffering. When it comes to relieving the suffering associated with chronic pain, a strictly biomedical approach is not only limited but at best not comprehensive and at worst potentially dangerous. As an evidence-based approach to chronic pain that exemplifies the biopsychosocial approach, TCM should be strongly considered for delivering truly patient-centered care.

\section{Patient-Friendly Recap}

- Current biomedical approaches to managing chronic pain are associated with high failure rates and a recent spike in narcotics abuse.

- The authors found growing evidence that acupuncture or tai chi, two forms of traditional Chinese medicine (TCM), can be used to effectively treat chronic pain.

- Given its overall cost-effectiveness, physicians should consider TCM as systematic or adjunct therapy to alleviate their patents' chronic pain. 


\section{Conflicts of Interest}

None.

\section{References}

1. Committee on Advancing Pain Research, Care, and Education; Board on Health Sciences Policy, Institute of Medicine. Relieving Pain in America: A Blueprint for Transforming Prevention, Care, Education, and Research. Washington, DC: The National Academies Press, 2011.

2. International Association for the Study of Pain: IASP taxonomy. http://www.iasp-pain.org/Taxonomy\#Pain. Accessed September 9, 2015.

3. American Academy of Family Physicians. Pain management and opioid abuse: a public health concern — executive summary (July 2012). http://www.aafp.org/dam/AAFP/documents/ patient_care/pain_management/opioid-abuse-position-paper. pdf. Accessed September 9, 2015.

4. Bruns D, Disorbio JM. Chronic pain and biopsychosocial disorders. Pract Pain Manag. 2005;5(7):2-9.

5. Gallagher RM. Biopsychosocial pain medicine and mind-brain-body science. Phys Med Rehabil Clin N Am. 2004;15:855-82. CrossRef

6. Goodman CC, Fuller KS (eds). Pathology: Implications for the Physical Therapist, Third Edition. St. Louis, MO: Saunders Elsevier, 2009.

7. Linton SJ, Shaw WS. Impact of psychological factors in the experience of pain. Phys Ther. 2011;91:700-11. CrossRef

8. Jamison RN, Virts KL. The influence of family support on chronic pain. Behav Res Ther. 1990;28:283-7. CrossRef

9. Ferreira PH, Ferreira ML, Maher CG, Refshauge KM, Latimer J, Adams RD. The therapeutic alliance between clinicians and patients predicts outcome in chronic low back pain. Phys Ther. 2013;93:470-8. CrossRef

10. National Center for Complementary and Integrative Health. Traditional Chinese medicine. https://nccih.nih.gov/health/ whatiscam/chinesemed.htm. Accessed September 10, 2015.

11. Kaptchuk TJ. The Web That Has No Weaver: Understanding Chinese Medicine, Second Edition. New York, NY: Contemporary Books (McGraw-Hill), 2000.

12. Wisneski LA, Anderson L. The Scientific Basis of Integrative Medicine. Boca Raton, FL: CRC Press, 2005.

13. Pomeranz B, Berman B. Scientific basis of acupuncture. In: Stux G, Berman B, Pomeranz B (eds). Basics of Acupuncture, Fifth Edition. Berlin, Germany: Springer, 2003, pp. 7-86.

14. Mi HF. The Systematic Classic of Acupuncture and Moxibustion, First Edition. Boulder, CO: Blue Poppy Press, 1994.

15. NIH Consensus Development Program, Office of Disease Prevention. Acupuncture. NIH Consensus Statement Online. 1997;15(5):1-34.

16. Hempel S, Taylor SL, Solloway MR, et al. Evidence map of tai chi. VA Evidence-Based Synthesis Program Reports. Washington, DC: U.S. Department of Veterans Affairs, September 2014.

17. Vickers AJ, Cronin AM, Maschino AC, et al. Acupuncture for chronic pain: individual patient data meta-analysis. Arch Intern Med. 2012;172:1444-53. CrossRef

18. Asher GN, Jonas DE, Coeytaux RR, et al. Auricular therapy for pain management: a systematic review and meta-analysis of randomized controlled trials. J Altern Complement Med. 2010;16:1097-108. CrossRef
19. Selfe TK, Taylor AG. Acupuncture for osteroarthritis of the knee: a review of randomized, controlled trials. Fam Community Health. 2008;31:247-54. CrossRef

20. Hopton A, MacPherson H, Keding A, Morley S. Acupuncture, counseling or usual care for depression and comorbid pain: secondary analysis of a randomized controlled trial. $B M J$ Open. 2014;4:e004964. CrossRef

21. Rakel D (ed). Integrative Medicine. Philadelphia, PA: WB Saunders, 2003.

22. Wang C, Bannuru R, Ramel J, Kupelnick B, Scott T, Schmid $\mathrm{CH}$. Tai Chi on psychological well-being: systematic review and meta-analysis. BMC Complement Altern Med. 2010;10:23. CrossRef

23. Hall A, Maher C, Latimer J, Ferreira M. The effectiveness of Tai Chi for chronic musculoskeletal pain conditions: a systematic review and meta-analysis. Arthritis Rheum. 2009;61:717-24. CrossRef

24. Wang F, Lee EK, Wu T, et al. The effects of tai chi on depression, anxiety, and psychological well-being: a systematic review and meta-analysis. Int J Behav Med. 2014;21:605-17. CrossRef

25. Peng PW. Tai chi and chronic pain. Reg Anesth Pain Med. 2012;37:372-82. CrossRef

26. Lewis RA, Williams NH, Sutton AJ, et al. Comparative clinical effectiveness of management strategies for sciatica: systematic review and network meta-analysis. Spine J. 2015;15:1461-77. CrossRef

27. Mao JJ, Kapur R. Acupuncture in primary care. Prim Care. 2010;37:105-17. CrossRef

28. Tick H. Integrative pain medicine: a holistic model of care. Pain: Clinical Updates. 2014;22(2):1-6.

29. Ernst E. Acupuncture: what does the most reliable evidence tell us? J Pain Symptom Manage. 2009;37:709-14. CrossRef

30. Wayne PM, Kaptchuk TJ. Challenges inherent to t'ai chi research: part I-t'ai chi as a complex multicomponent intervention. J Altern Complement Med. 2008;14:95-102. CrossRef

31. Wayne PM, Kaptchuk TJ. Challenges inherent to t'ai chi research: part II-defining the intervention and optimal study design. J Altern Complement Med. 2008;14:191-7. CrossRef

32. Linde K, Witt CM, Streng A, et al. The impact of patient expectations on outcomes in four randomized controlled trials of acupuncture in patients with chronic pain. Pain. 2007;128:264-71. CrossRef

33. Fuentes J, Armijo-Olivo S, Funabashi M, et al. Enhance therapeutic alliance modulates pain intensity and muscle pain sensitivity in patients with chronic low back pain: an experiment controlled study. Phys Ther. 2014;94:477-89. CrossRef

34. Centers for Medicare \& Medicaid Services, The Center for Consumer Information \& Insurance Oversight. Affordable Care Act Implementation FAQs - Set 15: provider non-discrimination. https://www.cms.gov/CCIIO/Resources/ FAct-Sheets-and-FAQs/aca_implementation_faqs $15 . \mathrm{html}$. Accessed September 15, $201 \overline{5}$.

(C) 2015 Aurora Health Care, Inc. 